\title{
Edema pulmonar por presión negativa secundario a parálisis bilateral de cuerdas vocales en postoperatorio de tiroidectomía total
}

\section{Negative pressure pulmonary edema secondary to bilateral vocal cord paralysis after total thyroidectomy}

Margarita María Reyes Silva, MD(1); Alirio Rodrigo Bastidas Goyes, MD(2)

\section{RESUMEN}

El edema pulmonar por presión negativa es una condición grave que puede asociarse a parálisis aguda de cuerdas vocales bilateral, y requiere del conocimiento fisiopatológico y clínico que permita la realización de un correcto diagnóstico y la instauración del tratamiento adecuado. Se presenta el caso de una paciente con edema pulmonar por presión negativa asociado a cirugía de tiroides, con recuperación exitosa.

Palabras clave: tiroidectomía, cuerdas vocales, periodo postoperatorio, edema pulmonar, presión negativa.

\section{ABSTRACT}

Negative pressure pulmonary edema is a serious condition that can accompany acute bilateral vocal cord paralysis. Pathophysiologic and clinical knowledge is required for correct diagnosis and adequate treatment. We present the case of a female patient with negative pressure pulmonary edema associated with thyroid surgery, whose recovery was successful.

Keywords: thyroidectomy, vocal cords, postoperative period, pulmonary edema, negative pressure.

\section{INTRODUCCIÓN}

El edema pulmonar por presión negativa (EPPN) es una complicación debida a la obstrucción aguda o a la liberación de la obstrucción crónica de la vía aérea superior. Ocurre generalmente en el postquirúrgico inmediato de los pacientes que cursan con alguna de estas condiciones (1). Se ha informado una incidencia entre el $0,05 \%$ al $0,1 \%$ de todas las prácticas anestésicas y hasta del $11 \%$ de todos los pacientes que requieren una intervención activa para el manejo de obstrucciones de la vía aérea (2). Las complicaciones fatales pueden llegar a ser hasta del $40 \%$, de ahí, que tener presente su fisiopatología, los hallazgos diagnósticos para un reconocimiento temprano y las medidas para un tratamiento adecuado es crucial para mejorar la calidad en la atención de los sujetos que lo padecen (3-5). Se presenta

(1) Residente de Medicina Interna Nivel II. Universidad Militar Nueva Granada. Hospital Militar Central, Bogotá, Colombia.

(2) Medicina Interna - Neumología - Epidemiología Clínica. Hospital Militar Central, Bogotá, Colombia. 
un caso de EPPN asociado a parálisis bilateral de cuerdas vocales en una paciente en postoperatorio de tiroidectomía total, a fin de recordar los aspectos más importantes de esta condición, así como, la importancia de la prevención de las lesiones del nervio laríngeo recurrente y la necesidad de una vigilancia postquirúrgica cuidadosa en sujetos con factores de riesgo y cirugía de vías aerodigestivas (6).

\section{PRESENTACIÓN DEL CASO}

Paciente de género femenino, de 36 años de edad, con historia de cinco meses de disfonía y aparición de masa en región cervical anterior con ecografía de tiroides que mostró dos lesiones nodulares en la glándula tiroidea; la primera de $12 \times 16 \mathrm{~mm}$ de diámetro, localizada en la unión del istmo con lóbulo izquierdo, hipoecoica, de bordes bien definidos con calcificaciones en su interior y la segunda de $8 \times 11$ $\mathrm{mm}$ en localización antero-inferior del lóbulo izquierdo, ecogénica, de bordes regulares y con halo hipo ecoico compatible con bocio multinodular. El perfil hormonal tiroideo fue normal. En sus antecedentes médicos narró rinitis alérgica sin tratamiento; los síntomas relacionados con apnea del sueño, antecedentes de alteraciones anatómicas faciales y procedimientos quirúrgicos en cara y cuello fueron negativos.

Fue programada para hemi-tiroidectomía izquierda; en los estudios pre-operatorios la radiografía de tórax fue normal y los análisis de química sanguínea, el cuadro hemático y los tiempos de coagulación no mostraron alteraciones. En cirugía se encontró, además de los hallazgos ecográficos, glándula tiroidea con múltiples microquistes en el lóbulo derecho y adenomegalias en nivel VI, llevándose a tiroidectomía total con vaciamiento ganglionar central bajo la sospecha de proceso maligno tiroideo. Después de la extubación y durante el postoperatorio inmediato, tuvo dificultad respiratoria asociada a estridor laríngeo, agitación psicomotora y disminución progresiva en oximetría de pulso con uso de músculos accesorios de la respiración y estertores en ambos campos pulmonares, de ahí que requiriera reintubación oro traqueal y soporte ventilatorio, titulación de presión positiva al final de la expiración y traslado a la unidad de cuidado intensivo ( $\mathrm{UCl}$ ).

A su ingreso a UCl la presión arterial fue de $116 / 71 \mathrm{~mm} \mathrm{Hg}$, frecuencia cardíaca de 110 latidos por minutos, frecuencia respiratoria de 20 respiraciones por minuto, temperatura $36^{\circ} \mathrm{C}$, saturación de oxígeno del $100 \%$ con intubación orotraqueal fijada a $22 \mathrm{~cm}$ y soporte ventilatorio en SIMV + PS 12, PEEP 10, VC: $400, \mathrm{FIO}_{2} 70 \%$. El tubo tenía secreciones muco-sanguinolentas espumosas, en cuello herida transversal en región anterior cubierta, sin evidencia de sangrado activo, sin hematoma ni deformidades, los pulsos carotídeos estaban presentes y simétricos; a la auscultación cardíaca se encontraba con ruidos cardíacos rítmicos, sin soplos ni extratonos; a la auscultación pulmonar se hallaron estertores diseminados en ambos campos pulmonares, sin sibilancias; abdomen normal, extremidades sin edemas y llenado capilar menor a dos segundos y examen neurológico con efecto de sedación con RASS - 1 sin hallazgos de focalización.

Se realizó estudio complementario con electrocardiograma de doce derivaciones normal, gases arteriales con pH 7,33, $\mathrm{PCO}_{2} 40.9 \mathrm{~mm} \mathrm{Hg}, \mathrm{PO}_{2} 96,6$ $\mathrm{mm} \mathrm{Hg}, \mathrm{HCO}_{3} 21,5 \mathrm{~mm} \mathrm{Hg}, \mathrm{SO}_{2} 97 \%$, BE -4, lactato 1,7, AG 14,2, fracción inspirada de oxígeno 0,7 , electrolitos con sodio $138 \mathrm{meq} / \mathrm{L}$, potasio $4,38 \mathrm{meq} / \mathrm{L}$, cloro $102,3 \mathrm{meq} / \mathrm{L}$, calcio $8,1 \mathrm{meq} / \mathrm{L}$, magnesio 1,57 meq/L, albúmina $3,83 \mathrm{~g} / \mathrm{dL}$ y troponina ultrasensible $0,004 \mathrm{ng} / \mathrm{mL}$ (hasta 0,014 ). La radiografía de tórax corresponde a la figura 1 .

El ecocardiograma transtorácico reportó buena función sisto-diastólica biventricular con fracción de eyección del ventrículo izquierdo del $78 \%$ e insuficiencia mitral mínima, PsAP $25 \mathrm{~mm} \mathrm{Hg}$. Recibió tratamiento con dosis única de furosemida endovenosa, esteroide parenteral (dexametasona) y reposición hormonal y electrolítica acorde con su patología con L-tiroxina, gluconato de calcio, carbonato de calcio + vitamina D y calcitriol. La evolución clínica fue adecuada y la paraclínica con normalización del estado ácido-base, oxigenación y desaparición de infiltrados en la radiografía de tórax (figura 1 Panel C). Se realizó extubación exitosa y la nasofibrolaringoscopia posterior mostró inmovilidad de cuerdas vocales bilateral en posición paramediana (figura 2).

Después de 24 horas de observación postextubación fue trasladada a salas de hospitalización para rehabilitación fonoaudiológica. El reporte patológico de lóbulo izquierdo de tiroides comprobó carcinoma papilar de tiroides variante folicular sin invasión vascular, en contacto con la cápsula tiroidea y el músculo estriado peritiroideo libre de lesión, lóbulo derecho e istmo con tiroiditis linfocítica crónica de Hashimoto y ganglios libres de tumor. 


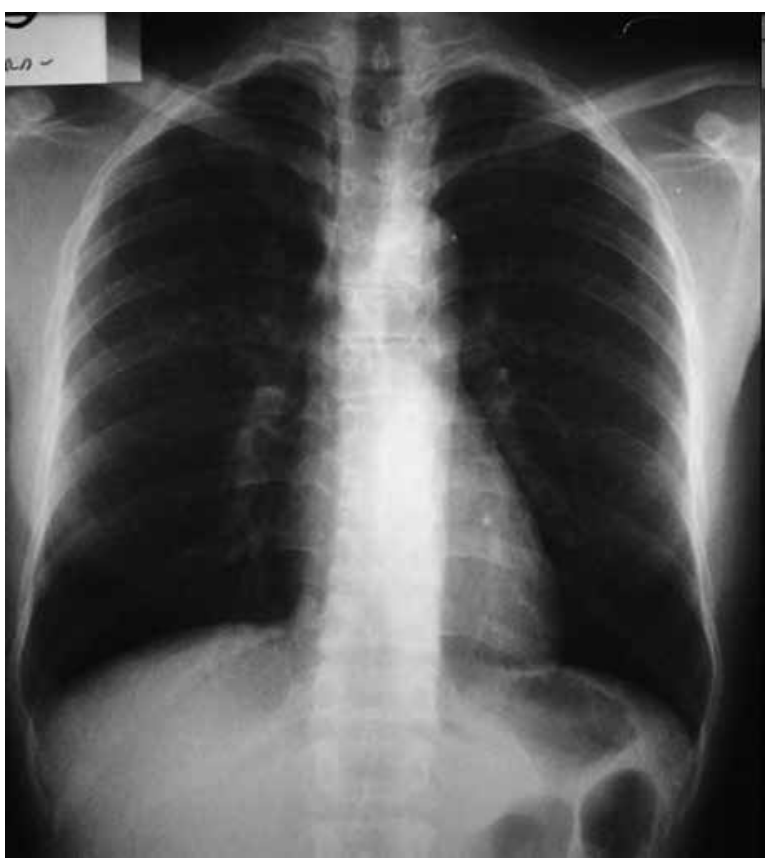

A.

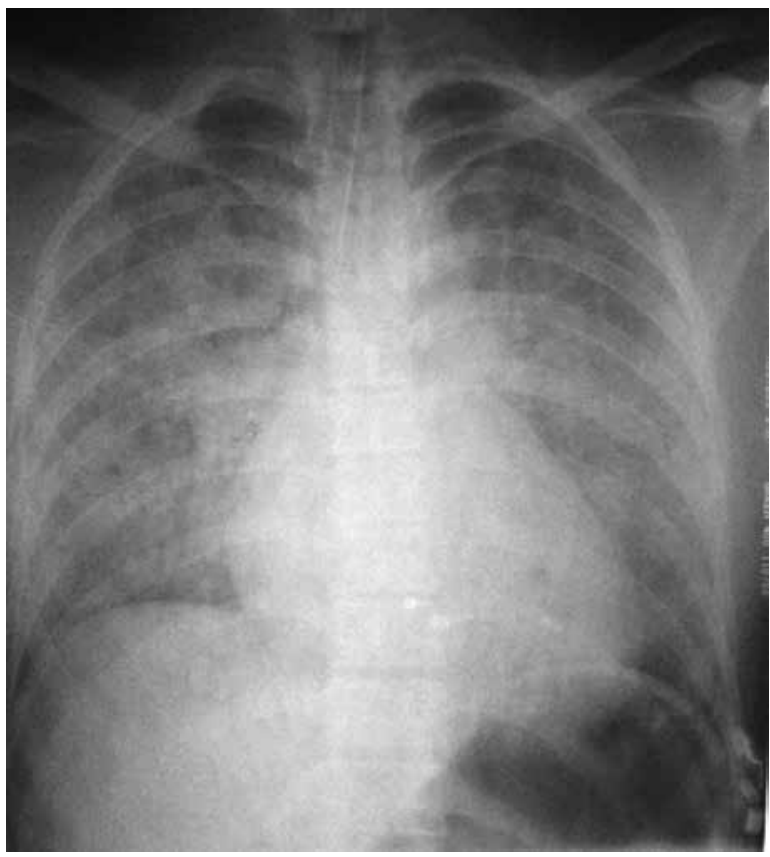

B.

Figura 1. Panel A: Radiografía posteroanterior de tórax antes de cirugía con parénquima pulmonar y silueta cardíaca normal; Panel B: radiografía antero posterior de tórax en post operatorio inmediato con imágenes de ocupación alveolar en cuatro cuadrantes, silueta cardio-mediastínica normal y visualización de tubo oro traqueal en tráquea a $4 \mathrm{~cm}$ de la carina, Panel C: Radiografía de tórax 24 horas posterior a ventilación mecánica con parénquima pulmonar con desaparición de infiltrado pulmonares.

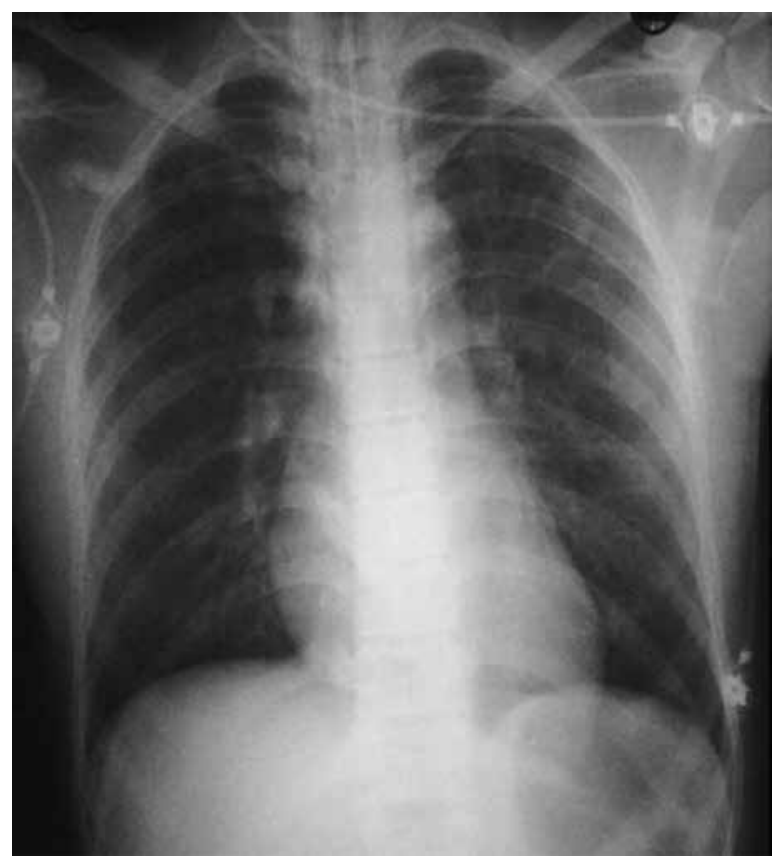

C.

\section{DISCUSIÓN}

Moore y Binger (7) describieron por primera vez el edema pulmonar por presión negativa en perros. De otra parte, en 1924 Warren describió la primera correlación fisiopatológica entre la presión negativa y el desarrollo de edema pulmonar; sin embargo, no fue sino hasta 1973 cuando Capitanio y Travis describieron la presencia de edema pulmonar en niños con epiglotitis y croup $(2,7)$. En 1977 Oswalt mostró por primera vez este fenómeno en adultos asociado a obstrucción aguda grave de la vía aérea (2) y posteriormente se han descrito múltiples casos asociados más frecuentemente a laringo-espasmo, así como también con otras condiciones como parálisis de cuerdas vocales (5), broncoespasmo severo (3), tumores de laringe, estrangulación, casi ahogamiento, mordedura de tubo endotraqueal durante la ventilación mecánica, posterior a drenaje de neumotórax o derrame pleural masivo, apnea del sueño, entre otras (4). Así mismo, se han descrito factores de riesgo que pueden estar asociados con la aparición de EPPN como: cuello corto, obesidad, acromegalia, índices elevados de intubación difícil, obstrucción mecánica de cánula orotraqueal, historia de apnea obstructiva del sueño, lesiones o anormalidades nasofaríngeas (8), individuos jóvenes con masa muscular acentuada (capaces de generar una presión negativa intra-pleural hasta $-100 \mathrm{~cm} \mathrm{H}_{2} \mathrm{O}$ ) y cirugía del tracto aerodigestivo (7). 

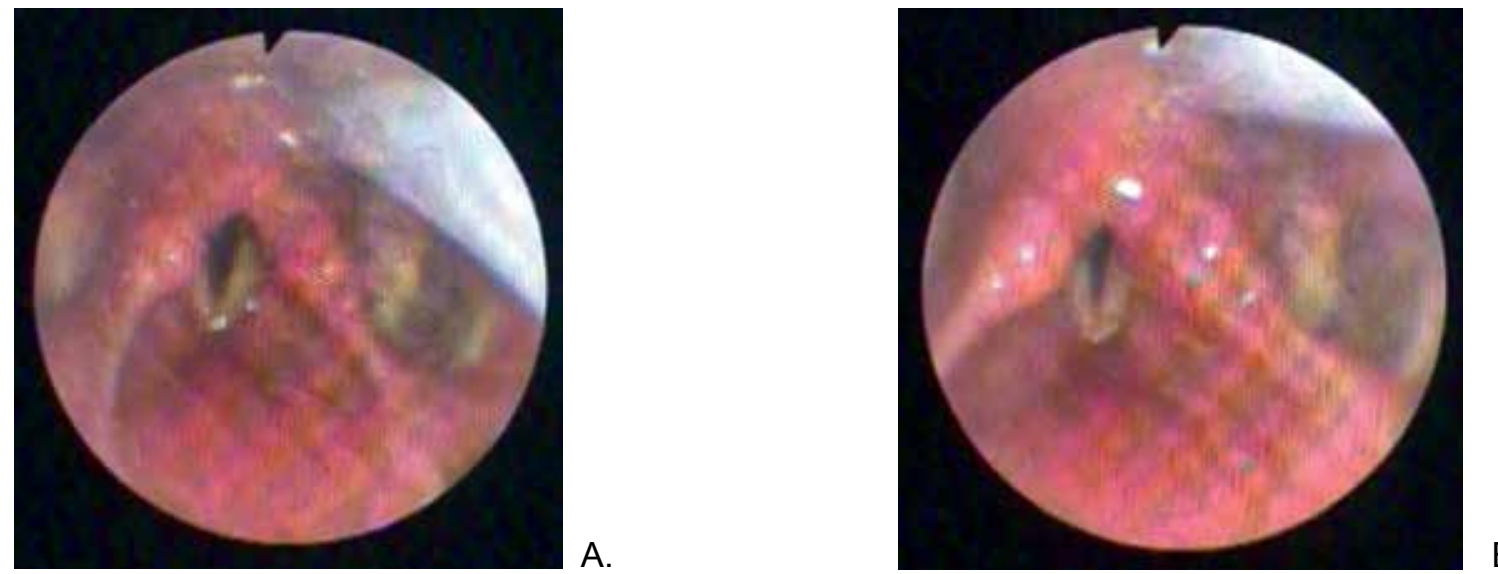

Figura 2. Fotos $A$ y B: parálisis de cuerdas vocales bilaterales en posición paramediana, cierre glótico incompleto sin lesiones en borde libre.

En el caso, la aparición abrupta de signos de dificultad respiratoria, estridor laríngeo y parálisis de cuerdas vocales asociados a secreciones rosadas espumosas en el tubo, estertores en ambos campos pulmonares e imágenes radiográficas en tórax de ocupación alveolar de rápida progresión en una paciente joven sin patología previa, sugiere el desarrollo de edema pulmonar por presión negativa (2, 4). La ausencia de hematomas expansivos en cuello o de evidencia clínica de reacción anafiláctica, y la demostración de una función cardiaca normal sin presencia de eventos isquémicos o arritmias, descarta otras posibles etiologías de edema pulmonar postoperatorio (4).

Desde la perspectiva fisiopatológica el edema pulmonar de presión negativa se considera no cardiogénico sin daño alveolar difuso (9), y se clasifica en dos tipos: tipo I asociado a obstrucción aguda de la vía aérea y tipo II secundario a liberación de la obstrucción crónica (7). En el EPPN tipo I inicialmente hay una obstrucción de la vía aérea que genera una negativización significativa de la presión intrapleural disminuyendo de -3 a $10 \mathrm{~cm}$ de $\mathrm{H}_{2} \mathrm{O}$ (rango normal) hasta $-50 \mathrm{a}-100 \mathrm{~cm}$ de $\mathrm{H}_{2} \mathrm{O}$ produciendo aumento del retorno venoso sistémico con elevación de las presiones capilares pulmonares. Al mismo tiempo y en relación con la interdependencia ventricular, hay incremento en la presión de fin de diástole del ventrículo izquierdo con disminución del retorno por las venas pulmonares a la aurícula izquierda, lo que lleva a la movilización rápida del fluido intra-vascular primero al intersticio y luego al espacio alveolar produciendo el edema pulmonar. Además, la hipoxemia secundaria a la obstrucción de la vía aérea lleva a un aumento de la resistencia vascular pulmonar y a la estimulación del sistema simpático con vasoconstricción periférica sistémica, que a su vez incrementa la postcarga del ventrículo izquierdo y aún más el volumen en el sistema vascular pulmonar, genera más trasudado en los espacios alveolares aumenta el shunt intrapulmonar y hay mayor hipoxemia (2). En los casos de obstrucción crónica de la vía aérea se genera un nivel de autopresión positiva al final de la expiración, con aumento del volumen pulmonar espiratorio final como medida compensatoria. Cuando se mejora la obstrucción, desaparecen súbitamente estas adaptaciones llevando a una presión intrapulmonar negativa que genera trasudación intersticial y alveolar configurando el EPPN tipo II $(2,4)$.

Las causas relacionadas con la aparición de EPPN están clasificadas según su fisiopatología; el laringoespasmo postextubación, la epiglotitis, el croup, el cuerpo extraño laríngeo, la estrangulación, la obstrucción del tubo endotraqueal, el tumor laríngeo, el bocio, el casi-ahogamiento, la succión directa de la cánula endotraqueal o adaptadores, la auto-extubación, la maniobra de Müller y la parálisis de cuerdas vocales se relacionan con el tipo I, mientras que la amigdalectomía o adenoidectomía, la exéresis de tumor en la vía aérea, la corrección de estenosis coanal, la resección de úvula hipertrófica redundante (7), la rino-septoplastia (10) entre otros procedimientos de vía aerodigestiva se asocian con el tipo II.

De otro lado, la incidencia de EPPN tipo I oscila desde $9,6 \%$ hasta $12 \%$ y en el tipo II es $44 \%$; el tipo 
I EPPN es más frecuente en individuos jóvenes, sanos y atléticos, con un ligero predominio masculino, mientras que el tipo II EPPN se ve en los extremos de las edades. La tasa de mortalidad global está entre el $10 \%$ al $40 \%(4)$.

La tiroidectomía a la cual fue llevada la paciente, es la causa más relacionada con parálisis aguda de las cuerdas vocales siendo este último un factor asociado a la aparición de EPPN. La frecuencia de parálisis de cuerda vocal en tiroidectomía oscila entre el $20 \%$ al $40 \%(11,12)$ y el compromiso bilateral entre el $0,5 \%$ al $5 \%$, porcentajes que varían según la extensión de la resección quirúrgica teniendo mayor riesgo de padecerla los sujetos llevados a cirugía por cáncer, enfermedad de Graves, re intervención y poca experiencia del equipo quirúrgico (6). El diagnóstico en este caso se realizó a través de nasofibrolaringoscopia y se puede complementar con electromiografía laríngea para confirmar la lesión del nervio laríngeo recurrente (12). En la paciente del caso, la parálisis de cuerda vocal fue en posición paramediana, que es la más descrita, pues se presenta hasta en un $78 \%$ y es superior a la parálisis en posiciones mediana e intermedia (11).

El diagnóstico de edema pulmonar por presión negativa es de exclusión, de ahí que deben descartarse otras causas de edema pulmonar postoperatorio como el edema cardiogénico y otros tipos de edema pulmonar no cardiogénico (neurogénico, sobrecarga de líquidos, anafilaxia, insuficiencia linfática (2) y SDRA (4). Se ha propuesto el gradiente albúmina líquido de edema y plasma para diferenciar el edema pulmonar hidrostático y el edema por aumento de la permeabilidad capilar; un gradiente menor de 0,65 sugiere edema hidrostático y uno mayor de 0,75 , indica edema pulmonar por permeabilidad aumentada (2).

El tratamiento se basa en la regulación de las presiones en la vía aérea, aunque adicionalmente en el EPPN tipo I se requiere aliviar la obstrucción según la causa subyacente para así mejorar la hipoxemia. La presión positiva en la vía aérea mediante ventilación mecánica invasiva o no invasiva es el pilar del tratamiento; además, pueden administrarse diuréticos a menos que el paciente esté hipovolémico, medidas suficientes en la mayoría de los pacientes con EPPN (2). Cuando hay estridor, el uso de corticosteroides ha mostrado reducción en el edema laríngeo y el requerimiento de reintubación (13). La evolución con desaparición de infiltrados pulmonares, con la instauración de presión positiva en la vía aérea y diurético en las primeras 48 horas como se describe en el caso, es el curso habitual de los edemas pulmonares no cardiogénicos asociados con cambios de presión; aunque algunos tardan más en recuperarse este retraso generalmente está asociado con diagnóstico y tratamiento tardíos (4).

No hay intervención demostrada que prevenga el EPPN, pero se ha planteado el beneficio de algunas medidas dirigidas a evitar las causas, como la irritación de la laringe (2), el uso de relajante muscular intra-operatorio, lidocaína tópica o inhalada, el despeje de secreciones en la vía aérea, el uso profiláctico de esteroides (13), la utilización de C-PAP en pacientes de alto riesgo (14) y, como en el caso expuesto, la identificación y preservación del nervio laríngeo recurrente por el cirujano con monitorización por electromiografía de estimulación laríngea $(1,6)$.

\section{CONCLUSIÓN}

El edema pulmonar por presión negativa es una complicación grave que puede ocurrir en pacientes con parálisis aguda de cuerdas vocales asociada a cirugía de tiroides. Por ende, es necesario que el equipo médico (cirujanos, anestesiólogos e intensivistas) conozcan los factores de riesgo, la fisiopatología, el diagnóstico y el tratamiento oportuno para lograr recuperaciones exitosas y disminuir el riesgo de complicaciones y mortalidad.

\section{BIBLIOGRAFÍA}

1. Kim WH, Ryu S a. Acute pulmonary edema secondary to upper airway obstruction by bilateral vocal cord paralysis after total thyroidectomy -A case report-. Korean J Anesthesiol. 2012; 62: 387-90.

2. Bhaskar B, Fraser JF. Negative pressure pulmonary edema revisited: Pathophysiology and review of management. Saudi J Anaesthesia [Internet]. 2011; 5: 308-13.

3. Krodel DJ, Bittner E a, Abdulnour R-EE, Brown RH, Eikermann M. Negative pressure pulmonary edema following bronchospasm. Chest. 2011; 140: 1351-4.

4. Bajwa SS, Kulshrestha A. Diagnosis, prevention and management of postoperative pulmonary edema. Ann Med Health Sciences Research. 2012; 2: 180-5.

5. Kim WH, Ryu SA. Acute pulmonary edema secondary to upper airway obstruction by bilateral vocal cord paralysis after total thyroidectomy -A case report-. Kor J Anesthesiol. 2012; 62: 387-90.

6. Aissaoui Y, Kichna H, Boughalem M, Drissi Kamili N. Acute negative pressure pulmonary edema: illustration from a unusual clinical case following a postoperative recurrent laryngeal 
nerve palsy. Ann Françaises d'Anesthèsie et de Rèanimation. 2012; 31: 481-3.

7. Orantes C, López SC, Ortiz JG. Edema agudo de pulmón por presión negativa, reporte de caso y revisión de literatura. Medicrit Revista De Medicina Interna y Crítica. 2008; 5 (3): 1037.

8. Demaría DA. Edema pulmonar a presión negativa. Una complicación anestésica. Rev Argent Anestesiol. 2008; 66: 17381.

9. Revelly J, Chiolero R, Vock P. Clinical and radiologic learning Radiographics. 1999; 19: 1507-31.

10. García de Hombre AM, Cuffini A, Bonadeo A. Negative pressure pulmonary oedema after septoplasty. Acta Otorrinolaringologica Espanola. SEGO; 2012; 2011-3.
11. Ahmad S, Muzamil A, Lateef M. A Study of incidence and etiopathology of vocal cord paralysis. Indian journal of otolaryngology and head and neck surgery : official publication of the Association of Otolaryngologists of India. 2002; 54: 294-6.

12. Pinto JA, Godoy LBDM, Marquis VWP, Sonego TB, Leal CDFA. Bilateral vocal fold immobility: diagnosis and treatment. Brazilian Journal of Otorhinolaryngology. 2011; 77: 594-9.

13. McCaffrey J, Farrell C, Whiting P, Dan A, Bagshaw SM, Delaney AP. Corticosteroids to prevent extubation failure: a systematic review and meta-analysis. Intensive Care Medicine. 2009; 35: 977-86.

14. Pathak V, Rendon ISH, Ciubotaru RL. Recurrent negative pressure pulmonary edema. Clinical Medicine \& Research. 2011; 9: 88-91. 